\title{
La pretendida curación de la escrófula por el toque del rey
}

\author{
Ignacio Duarte
}

\section{The pretended healing of scrofula by the king's touch}

From the $11^{\text {th }}$ century up to the beginning of the $19^{\text {th }}$ century a healing rite was performed by the kings of France and England. They were considered to have a hereditary divine power to cure with their hand the scrofula, a tuberculous lymphadenitis that affects mainly cervical lymph nodes. The rite took place regularly over groups of scrofulous patients and a wide audience. The belief in that miraculous power was based on the fact that kings had been anointed and crowned in a religious ceremony, thus acquiring a priestly nature together with their temporal power. The monarchs of France and England would have stimulated their subjects' credulity to strengthen their power over the feudal lords, specially when a change of dynasty took place. Scrofula may have been chosen due to a high incidence, with an evolution that may mimick healing, and also because the concept of scrofula may have included other lesions with episodes of spontaneous remission. The available historical data and the current knowledge of tuberculous lymphadenitis do not support the belief of massive miraculous healings by the king's touch.

Key words: Scrofula, lymphadenitis, tuberculosis, king's evil.

Palabras clave: Escrófula, linfoadenitis, tuberculosis, mal del rey.

\author{
Pontificia Universidad Católica \\ de Chile, Santiago, Chile. \\ Facultad de Medicina. \\ Departamento de Anatomía \\ Patológica. \\ Programa de Estudios Médicos \\ Humanísticos.
}

Declaración de conflictos de interés: Sin conflictos de interés.

Fuente de financiamiento:

presupuesto corriente del Programa.

Recibido: 9 de mayo de 2014

Correspondencia a: Ignacio Duarte García ignacioduarte.gc@gmail.com
$\mathrm{E}$ n la Edad Media se inició y desarrolló en Francia e Inglaterra un rito en el curso del cual se pretendía que el soberano, dotado de un poder milagroso, curaba a los enfermos de escrofulosis por el hecho de colocarles su mano mientras decía unas palabras piadosas.

Escrófula o escrofulosis era el nombre con que desde la antigüedad se designaba a una enfermedad caracterizada por aumento de volumen principalmente de los ganglios linfáticos del cuello, la cual a fines del siglo XIX y comienzos del siglo XX se identificó como linfoadenitis infecciosa tuberculosa.

En el estudio sobre la génesis y desarrollo de aquella pretensión sanadora de los reyes se ha podido apreciar la confluencia de varios factores que le dieron entidad a través de los siglos: documentos históricos, relatos de crónicas y biografías, leyendas transmitidas por generaciones, propaganda por móviles políticos y, sobre todo, la credulidad de la gente en una época en que se desconocían explicaciones científicas en el terreno de la salud y la enfermedad.

El objeto de este artículo es reseñar en primer lugar el desarrollo del rito del toque del rey. En segundo término, intentar responder las interrogantes acerca de por qué este poder habría sido detentado por reyes, en particular los de Francia e Inglaterra, y por qué se circunscribió a la curación de las escrófulas. Finalmente se concluirá con el examen de la decadencia y extinción del rito.

\section{Antecedentes}

En el medievo era frecuente que se atribuyera a hombres o mujeres virtuosos, vivos o muertos, o incluso a

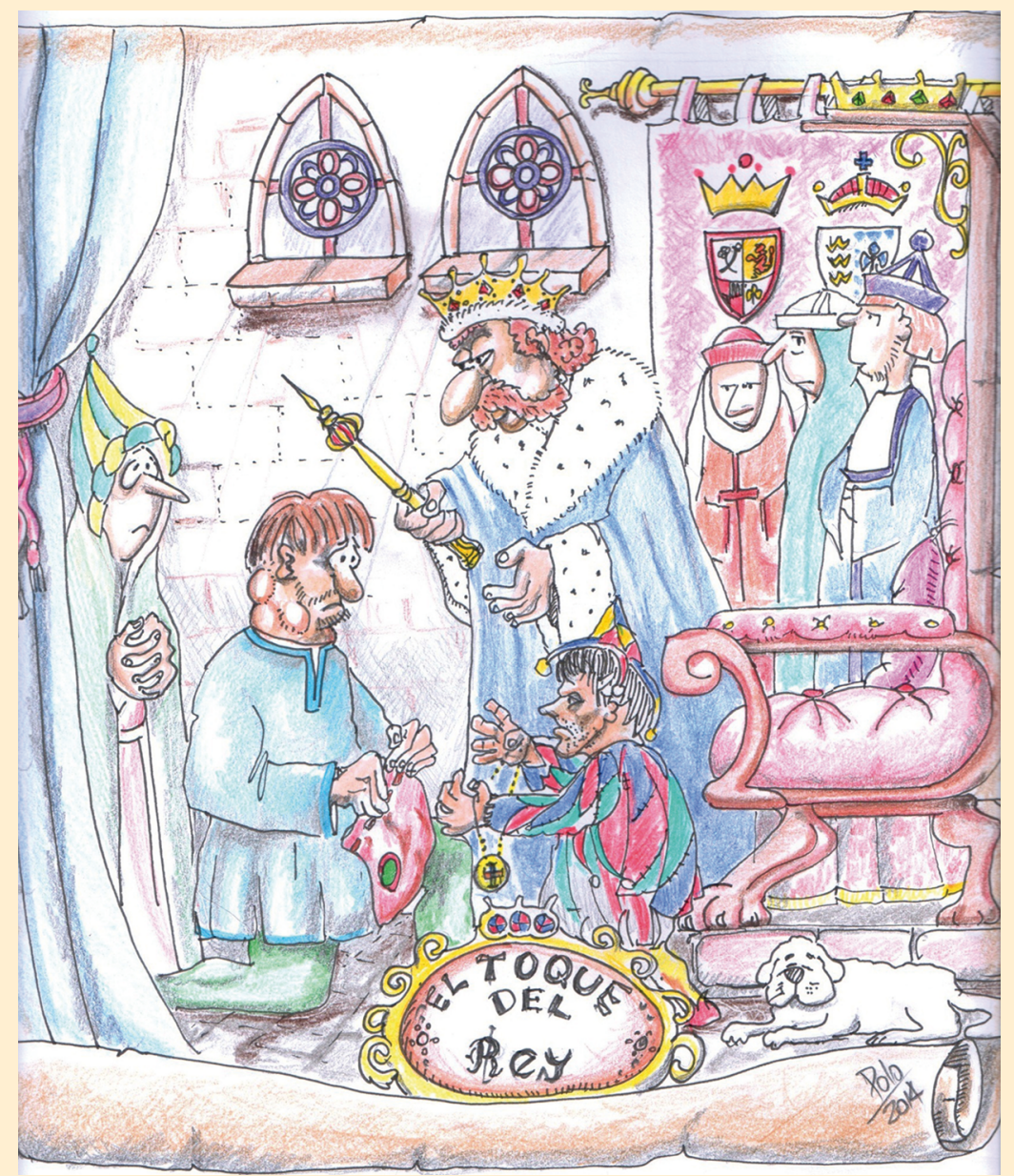

www.sochinf.cl 
sus reliquias, el poder de curar enfermedades. En algunos casos este carácter taumatúrgico se manifestaba de preferencia frente a determinadas enfermedades. Por ejemplo, se acudía a Santa Apolonia para el dolor de muelas o a San Antonio para las erisipelas ${ }^{1}$.

A fines del siglo X y comienzo del XI empezó a difundirse la fama de dos reyes considerados santos y milagrosos. Roberto II el Piadoso, segundo rey de la dinastía de los Capeto ocupó el trono en Francia desde 996 a 1031. La figura equivalente en Inglaterra fue Eduardo el Confesor, de la Casa de Wessex, que reinó desde 1042 a 1066.

Según el panegírico escrito alrededor de 1042 por el monje Helgaud de Fleury, contemporáneo y amigo de Roberto el Piadoso, este rey estaba adornado de numerosas virtudes, entre ellas bondad, misericordia y preocupación por los pobres. Se acercaba solícito a los enfermos, particularmente a los leprosos, les daba limosna y les besaba las manos. Se le había concedido la gracia de sanar las llagas con su piadosa mano ${ }^{2}$.

En 1066 o poco después se escribió un relato del primer milagro de Eduardo el Confesor en el caso de una joven que sufría de un aumento de volumen de las partes ubicadas debajo de la mandíbula, "llamadas glándulas". Le desfiguraba el rostro y exhalaba mal olor. Alertada por un sueño, recurrió al rey que no dudó en ayudarla. Mojó sus manos en un recipiente con agua y con la punta de sus dedos lavó y presionó repetidamente las partes afectadas, haciendo el signo de la cruz. En las zonas así masajeadas salieron "gusanos", pus y sangre por varios orificios. El rey persistió con el procedimiento hasta que se eliminó la enfermedad ${ }^{3}$.

\section{Desarrollo del rito}

En base a los documentos disponibles, los historiadores proponen que la serie de personajes que ejercieron el pretendido poder de curación hereditario de la realeza fue iniciada en Francia por Felipe I que gobernó desde 1060 a 1108 . No ha sido posible precisar cuáles de los más de 30 personajes que después detentaron el cetro francés hasta fines del siglo XVIII realizaron el rito de curación. Existe base para pensar que lo hicieron en los primeros tiempos Felipe I (1060-1108), Luis VI el Gordo (1108-1137), Luis IX el Santo (1226-1270), Felipe IV el Hermoso (1285-1314), Luis X el Obstinado (1314-1316) y después otros, algunos de los cuales se nombrarán.

Se ha sugerido que Enrique I que reinó desde 1031 a 1060 fue el iniciador de la serie en Inglaterra, habiéndose documentado que después protagonizaron la ceremonia, en los primeros tiempos, Enrique II (1154-1189), Eduardo I (1272-1307) y Eduardo IV (1461-1483) y otros. Igual que en el caso de Francia, los investigadores no han reunido datos suficientes para asegurar el grado de continuidad o discontinuidad del rito por parte de la treintena de personajes que rigieron Inglaterra hasta comienzos del siglo XIX ${ }^{4}$.

William Shakespeare menciona en su drama Macbeth el poder regio de curar en la siguiente escena protagonizada por Malcolm que había huido a Inglaterra después que Macbeth asesinó a su padre Duncan, rey de Escocia 5 .

MALCOLM: ...Por favor, ¿va a salir el rey?

MÉDICO: Sí, señor; hay una turba de infelices que esperan de él su curación. Su enfermedad desafia todos los esfuerzos del arte; mas, en cuanto les toca tal es la santidad que el Cielo ha concedido a su mano, se restablecen inmediatamente...

MACDUFF: ¿De qué dolencia se trata?

MALCOLM: La llaman el Mal (refiriéndose al "mal del rey") ${ }^{6}$. Es una cura milagrosa de este virtuoso príncipe, que varias veces, desde que vine a Inglaterra, se la he visto hacer. De cómo se entiende con el Cielo, mejor lo sabe él que nosotros; pero personas atacadas de extrañas dolencias, hinchadas y cubiertas de úlceras que daba lástima verlas, desahuciadas de la Medicina, las cura colgándoles del cuello una medalla de oro, mientras recita piadosas oraciones. Se dice que llegará a los reyes que le sucedan este sagrado poder de curar, con otra rara virtud: la del don celeste de la profecía, y las muchas bendiciones que rodean su trono nos hablan de hallarse en estado de gracia.

El editor de la versión de Oxford Shakespeare confirma que el dramaturgo se inspiró en la versión de 1587 de la Crónica de Escocia de Raphael Holinshed, en que se resalta el poder milagroso de Eduardo El Confesor quien acogió al refugiado Malcolm según el argumento de la tragedia ${ }^{7}$.

La expresión "mal del rey" (morbus regius) se había usado en la antigüedad y parte de la Edad Media para designar sucesivamente la ictericia, la lepra y afecciones que desfiguraban la apariencia del paciente ${ }^{3}$. Isidoro de Sevilla en el siglo VII mencionaba que algunos creían que la ictericia se llamaba mal del rey porque se curaba fácilmente con vino y comidas dignas de reyes $^{8}$. En el siglo XIII "mal del rey" se comenzó a usar respecto a la escrófula en el sentido de que era la enfermedad curable por el rey. El acto por el cual este ponía su mano con intención curativa sobre el enfermo pasó a llamarse el tacto o toque del rey.

En el principio, el tacto se hacía a personas afectadas de diversos males, pero después se circunscribió a aplicarlo solamente a las escrófulas. Se ha discutido si el primero que tocó exclusivamente escrofulosos en Francia fue Felipe I o Luis IX; en el caso de Inglaterra, si fue iniciado por Enrique I, Enrique III o el hijo de éste, Eduardo I ${ }^{3,9}$.

El rito del toque del rey fue evolucionando desde un acto simple al que se fueron agregando otros componentes 
para llegar a ser una ceremonia más compleja, con algunas diferencias entre Francia e Inglaterra, pero en síntesis llegó a estar constituido por la siguiente secuencia:

Los médicos de la corte seleccionaban con anticipación a los escrofulosos, descartando a los afectados por otro mal. El rey se preparaba, a veces ayunando en el día previo. El rito mismo se iniciaba con la celebración de la misa, en que el rey comulgaba. Después de la misa se acercaba a los pacientes escogidos: a cada uno tocaba la cara o el cuello, hacía sobre él la señal de la cruz y rezaba una corta oración o le decía algunas palabras. A continuación el capellán del rey entregaba a algunos, especialmente a los que venían de lejos, una limosna. Finalmente se leían algunos pasajes del Evangelio, en particular el párrafo en que Jesús dice a sus discípulos que "pondrán la mano sobre los enfermos y se pondrán bien" $" 10$.

Eduardo IV de Inglaterra mandó acuñar una moneda de oro que tenía un grabado del Arcángel Miguel matando al dragón, símbolo del demonio: por eso se empezó a llamar "ángel" a la moneda, que se entregaba a los enfermos como limosna ${ }^{11}$. Tiempo después se procedió a perforarlas y agregarles una cinta con la que se les colgaba alrededor del cuello, como aparece en la escena de Macbeth. El diseño de la medalla cambió a través de algunas generaciones; también hubo una época en que se confeccionaron en plata. A los enfermos se les recomendaba que conservaran siempre consigo el "ángel", porque era un elemento indispensable de la fórmula de curación.

La oportunidad en que se realizaba el rito de curación variaba. En algunos casos el rey la estrenaba con motivo de su coronación, o bien durante alguna fiesta religiosa como la Pascua de Resurrección, Pentecostés, Conmemoración de todos los Santos, Natividad de Jesús, Fiesta de San Miguel Arcángel. Luis IX solía practicarlo a pedido de los enfermos después que asistía a misa. Carlos II de Inglaterra tocó enfermos incluso durante su exilio en Europa continental ${ }^{12}$. En ciertas ocasiones la ceremonia se suspendía, por ejemplo durante viajes de los reyes, o por la costumbre de Carlos II y Jacobo II de Inglaterra de no realizar la ceremonia en verano, por temor a que las enfermedades agudas estacionales se esparcieran entre los asistentes ${ }^{13}$.

No era pequeño el número de escrofulosos atendidos. Por ejemplo, en Francia Luis XIII a los 9 años de edad tocó a 450 enfermos en una sesión, y Luis XVI a unos 2.400 tres días después de su coronación. En Inglaterra Eduardo III tocó a 885 enfermos en alrededor de dos años, y Carlos II a un total de 92.102 en 22 años $^{14}$.

André du Laurens, médico de Enrique IV de Francia, publicó en 1609 el libro "La admirable cura de la escrófula, poder concedido sólo a los cristianísimos reyes de Francia" Algunos ejemplares incluían un grabado de Pierre Firens que ha llegado a constituir una de las más populares representaciones de la ceremonia. En primer plano Du Laurens sujeta firmemente la cabeza de un escrofuloso, que está arrodillado. El rey toca al paciente. El confesor del rey aparece detrás de éste, posiblemente distribuyendo la limosna. Los demás enfermos están arrodillados, distribuidos en semicírculo, respaldados por guardias armados que evitan la irrupción de la multitud que se encuentra al fondo, algunos tal vez procurando sumarse al grupo de pacientes que esperan la curación (Figura 1) ${ }^{15}$.

En Inglaterra John Evelyn relata en su diario que asistió a la ceremonia del toque del Rey Carlos II en 1660. Esta se llevaba a cabo en el Palacio de Banquetes de Whitehall. Evelyn anota lo siguiente:

...Su Majestad se sentó bajo su baldaquín: el cirujano hizo traer al primer enfermo, lo condujo hasta el trono, delante del cual se hincó. El rey le tocó la cara con ambas manos. El capellán ceremoniosamente recitó: Él puso sus manos sobre ellos y los curó. Cuando todos los solicitantes habian sido tocados, se acercaron nuevamente en el mismo orden. El otro capellán, de rodillas y con los ángeles de oro colgados de una cinta blanca en su brazo, se los entregó uno por uno al rey, quien los colocó al cuello de cada enfermo a medida que pasaban, mientras el primer capellán repetia: Esta es la verdadera luz que vino al mundo. Siguió la lectura de la Epístola-antes se habia leido el Evangelio- y las oraciones litúrgicas por los enfermos. Finalmente se dio la bendición y entonces el chambelán trajo un recipiente, jarra y toalla para que el rey se lavara ${ }^{16}$.

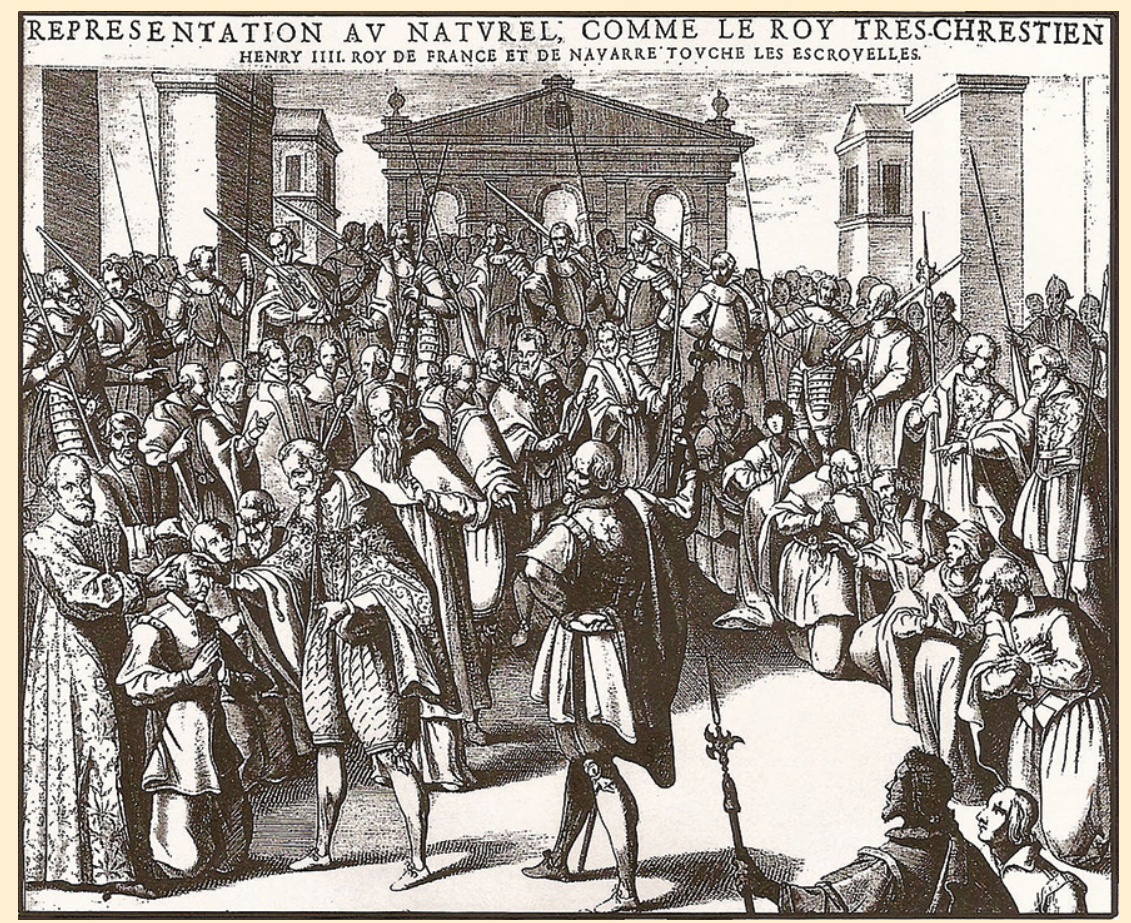

Figura 1. Scrofula. André du Laurens, 1609. 


\section{¿Por qué los reyes?}

Hasta el siglo XI el gobierno de los monarcas no se había afianzado adecuadamente. El rey era un primum inter pares, en este caso entre los nobles. No tenía control efectivo sobre lo que sucedía en las regiones, donde la autoridad de duques o condes primaba sobre la autoridad central por la falta de un sistema burocrático eficiente que manejara aspectos políticos, económicos y administrativos. No se había desarrollado un concepto dinástico.

La situación se empezaba a modificar paulatinamente: en el siglo XII se iba fortaleciendo el gobierno central. El monarca tendía a permanecer en una sede, se desarrollaron instituciones, procesos y formación de funcionarios administrativos, lo que redundó en mayor uso de la escritura y generación de documentos y archivos. Se profundizó el estudio y aplicación del Derecho, entre cuyos temas se suscitaron análisis sobre el ejercicio del poder.

El fortalecimiento de la posición de los monarcas frente a sus súbditos en general y a los nobles en particular se sustentaba en una base teológica con elementos jurídicos y políticos ${ }^{17}$. El soberano, por la gracia de Dios, era una imagen de Cristo. Encarnaba la justicia de Dios en la tierra. Además, en su persona, incorporaba el cuerpo político del reino, formado por él y todos los miembros o súbditos, cada uno con su propia dignidad. En consecuencia, el rey debía ser piadoso, protector y defensor de la Iglesia, legislador, buen juez, líder militar, protector de los necesitados y hábil en tratar con los nobles para obtener su consejo y apoyo.

Según la teoría teológica, el monarca recibía la gracia especial de Dios como efecto de la ceremonia de su consagración, ceremonia que daba inicio oficial a su reinado. La consagración constaba de dos elementos esenciales: la unción con el crisma y la coronación. La unción consistía en derramar el aceite bendecido previamente en una ceremonia especial y que tenía el significado de conferir carácter sacerdotal o de realeza a quien lo recibía. Esto puede apreciarse en relatos del Antiguo Testamento como aquel en que Moisés derramó aceite sobre la cabeza de Aarón para consagrarlo sacerdote ${ }^{18}$, o cuando David y Salomón fueron ungidos reyes ${ }^{19,20}$.

En Europa había antecedentes de la unción aplicada a monarcas, por ejemplo a los reyes visigodos. En 751 Pepino III el Breve había sido ungido rey de los Francos en Soissons por el obispo Bonifacio. Cuando Carlomagno fue solemnemente proclamado emperador en la basílica de San Pedro, el Papa León III, después de la unción, tomó la corona y la colocó en la cabeza del ungido, aunando así un carácter de tipo sacerdotal a la posesión de la insignia del poder temporal ${ }^{21}$.

La consagración del soberano quedó incorporada y constituía una ceremonia de gran solemnidad, como se sintetiza aquí en el en el relato de la entronización en 1189 de Ricardo II Corazón de León en Inglaterra:

Un gran séquito de obispos, abades, clero, barones, condes y duques precedidos por la cruz y portadores de velas, incensarios y agua bendita escoltaron en procesión al duque Ricardo desde su cámara hasta el altar mayor de la iglesia de Westminster. Se hincó frente al altar y juró por los Evangelios observar la paz, el honor y la reverencia hacia Dios, la Santa Iglesia y sus ordenanzas. Juró también ejercitar la verdadera justicia y equidad hacia el pueblo, abrogar las malas leyes y costumbres injustas y decretar buenas leyes, observándolas sin fraude o mala intención. El arzobispo de Canterbury, derramando santo crisma sobre su cabeza, lo ungió como rey en su cabeza, pecho y brazos, con las oraciones adecuadas para la ocasión. Lo vistieron con los mantos reales y se le entregó la espada de gobierno. Prometiendo observar los juramentos antes mencionados, él mismo tomó la corona del altar y se la dio al arzobispo, quien se la colocó. Comenzó la misa y, una vez terminada, fue acompañado en solemne procesión a su cámara, coronado y llevando el cetro en su mano derecha y la vara de realeza en la izquierda. Finalmente se celebró un gran banquete ${ }^{22}$.

La suma de la unción y la coronación pasó a ser la "consagración" de la realeza sagrada y el fundamento de la pretensión del poder milagroso que detentaban los monarcas, poder que heredaban sus sucesores reinantes ${ }^{23}$.

En el siglo XI existía cierta relación entre los dos poderes supremos de la cristiandad. En el caso de Alemania el rey era designado por los príncipes electores y coronado emperador por el papa; el emperador, a su vez, controlaba el buen orden de la elección pontificia. El papa y el emperador ejercían la autoridad suprema cada uno en su propio ámbito ${ }^{24}$.

El Papa Gregorio VII se propuso a partir de 1075 emprender una reforma, uno de cuyos objetivos principales era liberar a la Iglesia de la participación de los poderes civiles o laicos que imponían a sus candidatos invistiéndolos en cargos de obispo, abad u otra dignidad. Estos muchas veces no tenían formación ni interés para desempeñar las funciones religiosas correspondientes. Se desencadenó la llamada Querella de las Investiduras por la cual el poder espiritual -léase el papado- y el poder temporal representado por emperadores y reyes, no estaban dispuestos a perder sus prerrogativas ${ }^{25}$.

Como consecuencia de lo anterior, se empezaron a manifestar desacuerdos en cuanto al carácter sacerdotal y el poder taumatúrgico que la unción confería a los reyes. Así en el siglo XII Pierre de Blois, que fue secretario de Enrique II de Inglaterra, escribía:

Confieso que asistir al rey es cumplir una función santa; pues el rey es santo: es el Cristo del Señor. No en vano ha recibido el sacramento de la unción, cuya eficacia quedaría ampliamente demostrada por la desaparición de esa peste que ataca la ingle y por la curación de las escrófulas ${ }^{3}$. 
Por su parte el abate Guilbert de Nogent en Francia, que relataba haber sido testigo de la cura de las escrófulas por Luis VI, afirmaba que los milagros no son en sí manifestaciones de santidad, puesto que Dios es su único autor y puede canalizarlos a través de personas que convienen a sus designios aunque sean impíos ${ }^{26}$.

En el contexto de esta discordia entre los partidarios de la preeminencia del poder espiritual y los del poder temporal, los primeros repetían que el rey -que recibía la unción- era inferior al sacerdote que la confería.

Las consideraciones expuestas pueden haber influido en que las palabras piadosas que el rey recitaba junto con el tacto fuesen siendo reemplazadas por la fórmula "El rey te toca; Dios te cura", como se aprecia en la descripción del rito de Enrique IV en Francia ${ }^{27}$.

\section{¿Por qué Francia e Inglaterra?}

Desde fines del siglo $\mathrm{X}$ hasta el transcurso del siglo XII se produjeron en Francia e Inglaterra cambios de dinastías en el trono, lo que acentuó la necesidad de los nuevos gobernantes de afianzar su legitimidad.

En Francia, tras la muerte del último rey carolingio, el duque Hugo Capeto fue coronado rey en 987 con el apoyo de un grupo de nobles. La institución de la monarquía estaba muy debilitada: Hugo debió enfrentar las exigencias de privilegios de sus sostenedores y, por otra parte, la resistencia de otros duques, condes, y barones que lo consideraban un usurpador. Nombró como sucesor a su hijo Roberto el Piadoso, lo cual dio comienzo a la continuidad de la vigencia de la dinastía de los Capeto que, teniendo en consideración sus ramas, ocupó el trono hasta el siglo XVIII ${ }^{21}$. Dado que no hay datos de que el sucesor de Roberto, su hijo Enrique I de Francia haya obrado curaciones milagrosas, se ha sostenido que Felipe I, nieto de Roberto, fue el verdadero iniciador del pretendido poder milagroso hereditario exhibido en Francia, aunque algunos asignan a Roberto II el Piadoso el carácter de precursor o iniciador.

Tras vencer en la batalla de Hastings en 1066 el duque Guillermo de Normandía -llamado el Conquistador- fue coronado como primer rey de la dinastía normanda que sustituyó a la de la casa sajona de Wessex en Inglaterra. Fue sucedido por sus hijos Guillermo II y Enrique I: éste impuso paz y tranquilidad, tomó medidas para atraer a los sajones, por ejemplo casándose con una princesa de esa sangre, impulsando una justicia más imparcial y organizando el aparato administrativo del reino ${ }^{28}$. Según Bloch, Enrique I en su empeño por dar estabilidad y prestigio a la institución monárquica, adoptó y adaptó el rito francés asignándolo como originado en Inglaterra a partir del poder milagroso de Eduardo el Confesor ${ }^{29}$, como se sugiere en la escena de Macbeth. Dado que existe testimonio escrito de que Felipe I en Francia practicaba el rito - posiblemente en el último tercio del siglo XI- y que Enrique lo hacía en el primer tercio del siglo XII, ello indica una secuencia temporal compatible con esa hipótesis. Además Enrique I tenía fuertes vínculos con Francia por haber tenido un ducado en Normandía y haber permanecido unos años en aquella región incluso durante su reinado en Inglaterra ${ }^{30}$. Del mismo modo, existieron relaciones entre otros monarcas de Francia e Inglaterra que pueden explicar una mutua influencia en el desarrollo del rito en ambos reinos.

En otros países europeos se esparció la fama de casos aislados de reyes con poder milagroso de curar enfermedades. Esto, sin embargo, no se tradujo en una continuidad que se interpretara como un don divino heredable a las sucesivas generaciones de monarcas como se logró en Francia e Inglaterra.

\section{¿Por qué las escrófulas?}

Actualmente en países desarrollados la linfoadenitis tuberculosa representa 5 a $10 \%$ de los casos de tuberculosis activas ${ }^{14}$, aunque en grupos de inmigrantes puede alcanzar hasta $44 \%$. Es causada por un conjunto de especies de bacilos del género Mycobacterium relacionadas genéticamente, que han sido agrupadas en el Complejo de Mycobacterium tuberculosis. También pueden causar linfoadenitis otros bacilos del conjunto de micobacterias no tuberculosas ${ }^{31}$. Entre las del complejo de Mycobacterium tuberculosis hay dos que históricamente han afectado con mayor frecuencia a la humanidad. Una es Mycobacterium tuberculosis, descubierta por Koch, que característicamente asienta en los pulmones y se transmite por una persona enferma mediante gotas microscópicas expulsadas al aire en la tos y esputos, que son aspiradas por la persona sana. La otra especie es Mycobacterium bovis, bacilo bovino que suele afectar al ganado, desde donde infecta al ser humano cuando éste consume leche o carne contaminada, produciendo sus lesiones destructivas iniciales en la pared del tubo digestivo.

Se describen dos vías principales por las cuales los bacilos pueden provocar la linfoadenitis tuberculosa cervical. En una el punto de partida es un pulmón tuberculoso, donde los bacilos penetran en vasos sanguíneos, y mediante la circulación llegan a colonizar y dañar los ganglios. Alternativamente, cuando una persona se contagia por primera vez con el bacilo, por vía oral, aparece la primera lesión en la pared de la faringe, desde donde los bacilos son transportados en los vasos linfáticos a los ganglios del cuello.

El bacilo bovino ha sido una causa común de linfoadenitis cervical humana en países con alta prevalencia de tuberculosis del ganado. Esto puede explicar que en 
el pasado fuera frecuente la escrofulosis en comparación con la tuberculosis pulmonar, antes de que se empezara a extender en occidente en la primera mitad del siglo XX la eliminación del ganado enfermo y la pasteurización de la leche mediante la destrucción de sus gérmenes patógenos por un golpe de calor ${ }^{32}$. Se ha planteado que la linfoadenitis cervical producida por el bacilo bovino induce cierta protección contra la infección por $M$. tuberculosis, lo que puede apoyar la suposición de que hubiera una menor prevalencia de compromiso pulmonar en tiempos en que se consumía más leche contaminada ${ }^{14}$. $\mathrm{Al}$ respecto, es pertinente recordar que la vacuna $\mathrm{BCG}$ contra la tuberculosis fue obtenida por Calmette y Guérin por inactivación de la virulencia de una cepa de bacilo bovino mediante repetidos subcultivos ${ }^{33}$.

Más de trece siglos antes que Robert Koch en 1882 identificara bacilos en cortes de muestras de lesiones escrofulosas humanas ${ }^{34}$, el escritor médico Cassius Felix en el siglo V la caracterizaba así:

Las escrófulas son cuerpos redondeados implantados en los tendones, arterias, venas y membranas musculares [...] Cuando se desarrollan en las áreas glandulares a ambos lados de la garganta duelen, y sobresalen como el cuello abultado de una cerda: por eso se llaman scrofae. Son dificiles de curar. Se originan en formaciones ocupadas por un humor malsano. Hay también otra clase de escrófulas, no muy adheridas a la piel, como flotando. Se multiplican como cerdos: por eso, nuevamente, se llaman scrofae. Como dijimos, se originan en regiones glandulares como el cuello, la axila y la ingle. Estas se curan fácilmente, sea por cirugía o medicina ${ }^{3}$.

Se pueden plantear cinco posibles motivos por los cuales las escrófulas pasaron a ser objeto del toque.

El primero es la frecuencia de la afección, como parece desprenderse del altísimo número de personas que, seleccionadas por médicos de la época, accedían a la atención del rey. Se ha sugerido que los registros del número de personas tratadas son probablemente indicadores confiables de una alta prevalencia de la tuberculosis ${ }^{35}$ : llama, sin embargo, la atención la abundante mención de las escrófulas en el medievo en comparación con la muy escasa información de la época sobre la tuberculosis pulmonar $^{36}$, que ya había sido descrita en los Tratados Hipocráticos alrededor del siglo IV a.C.

El segundo motivo puede corresponder al hecho de que muchas veces la escrófula era difícil de curar por el arte médico, como lo expresaba Richard Wiseman a fines del siglo XVII calificándola "como una enfermedad contumaz que frecuentemente frustra los mejores cuidados y la destreza del cirujano" 37 . El rey milagroso representaba una última instancia con su presunto poder sobrenatural.

El tercer hipotético motivo de elección de las escrófulas para el rito estriba en dos posibles formas de evolución que pudieran interpretarse como curación: si correspondía a un complejo primario causado por bacilo bovino, el ganglio tumefacto podía curar por fibrosis y calcificación del componente ganglionar que disminuyera de tamaño hasta empequeñerse notoriamente o hacerse imperceptible. Si la lesión se ulceraba y fistulizaba, podía dar la apariencia de curación por cicatrización, como lo consignaba a principios del siglo XIX un famoso diccionario médico:

En la mayor parte de los individuos, los tumores, los abscesos y las úlceras escrofulosas nacen, desaparecen, y se suceden por espacio de mucho tiempo; dejan señales profundas en las partes afectadas, y cuando la naturaleza ayudada por el arte ha puesto término a esta larga serie de males, las infelices víctimas llevan por toda su vida las cicatrices indelebles de esta cruel enfermedad ${ }^{38}$.

El cuarto motivo o explicación apunta a que el concepto y diagnóstico de escrofulosis era impreciso ${ }^{39}$. Tumoraciones tocadas por el rey pueden haber correspondido a lesiones no tuberculosas con tendencia espontánea a involucionar. En el espectro de masas cervicales según su ubicación topográfica y otros caracteres semiológicos, microbiológicos y radiológicos no disponibles en aquella época, se pueden enumerar entidades de curso grave como neoplasias y linfoadenitis piógenas agresivas; pero también podía haber otras susceptibles de episodios de regresión como las producidas en los ganglios por linfoadenitis banales, hiperplasias linfoides reactivas, toxoplasmosis aguda o enfermedad de Kikuchi-Hashimoto. Alternativamente puede tratarse de quistes branquiales o tiroglosos, nódulos tiroideos, sialoadenitis o sialolitiasis.

Por último se debe considerar un favorable efecto psicológico de bienestar de los enfermos al experimentar una atención personalizada de su soberano en un ambiente solemne preparado para estimular la confianza en el procedimiento $^{27}$.

\section{Decadencia y extinción del rito}

Es innegable la popularidad del toque del rey, atestiguada por su duración durante siglos y los resultados descritos por testigos como Richard Wiseman, cirujano oficial de Carlos II de Inglaterra que dijo: "Yo mismo he sido frecuente testigo ocular de muchos cientos de curaciones logradas solamente con el toque de Su Majestad, sin ayuda de la cirugía" ${ }^{40}$. Sin embargo, lentamente fueron surgiendo dudas acerca del carácter milagroso y de la eficacia de la ceremonia.

Entre los factores que contribuyeron al descrédito del rito se puede mencionar en primer lugar la progresiva difusión del racionalismo que menguó en general la fe en lo sobrenatural y en particular la idea del origen divino de la monarquía, pasando lentamente a concebirse al rey sólo como un representante hereditario del Estado. En segundo lugar, las consecuencias de la Reforma protes- 
tante con la polarización religiosa, las actitudes de apoyo o rechazo de los reyes a alguna de las facciones, y en Inglaterra la hostilidad de los protestantes más radicales hacia el rito, tanto por razones doctrinarias como por la figura del rey que personificaba la responsabilidad por las persecuciones de que eran objeto. A estos factores debe agregarse el surgimiento de charlatanes y practicones que pregonaban su capacidad de sanar enfermedades, incluyendo las escrófulas; algunos de estos personajes operaban por generaciones en grupos familiares, tenían habilidad comercial y propagandística, y en ocasiones conseguían respaldo de autoridades ${ }^{41}$.

El escepticismo o duda sobre el mérito del toque del rey se manifestaba en forma más o menos sutil. Así el historiador Fuller en 1655 consideraba que un verdadero milagro opera al instante y de modo perfecto, mientras que aquella cura evolucionaba paulatinamente ${ }^{42}$. Por su parte Samuel Pepys después de asistir por primera vez a la ceremonia, realizada con gran solemnidad por el rey, concluyó en su diario que le había parecido un oficio desagradable e ingenuo (an ugly office and a simple one)" ${ }^{43}$. Wiseman se refiere a enfermos sanados que no atribuyeron su curación al rey, sino al ejercicio del viaje, al cambio de aire, a pura imaginación o al efecto de la medalla de oro ${ }^{44}$.

Hubo casos en que no había información acerca de si se había curado la escrófula. Du Laurens escribía que después del toque de Enrique IV los agudos dolores que sufren algunos disminuyen o desaparecen inmediatamente. En otros las úlceras se secan, y los tumores de otros disminuyen de tamaño, de tal modo que en pocos días más de 500 pacientes en 1.000 quedan completamente curados $^{27}$.

Por otra parte, se sabe de pacientes que recibieron el toque en más de una ocasión, y que se empezó a difundir la idea de que el toque no producía efecto si no se repetía. De alguna manera la sensación de recelo que crecía puede haber influido en el cambio de sentido de la frase "El rey te toca; Dios te cura" por la fórmula de Luis XV: "El rey te toca; que Dios te cure"27.

Una información del siglo XVII indica una de las posibles evoluciones que pueden haber experimentado pacientes tocados por el rey: El profesor de teología Miguel Martín de Portugal fue enviado desde Portugal a Francia para obtener la sanación de sus escrófulas mediante el tacto del cristianísimo rey. Regresó curado; sin embargo, posteriormente sucumbió de otro mal, víctima de una lenta consunción ${ }^{45}$. Puede pensarse que el paciente era víctima de una tuberculosis diseminada; pero no hay datos para explicar en qué habría consistido la desaparición de la escrófula.

En el caso de Inglaterra, existe documentación que permite afirmar que a partir del siglo XVI hasta fines del siglo XVII practicaron el tacto los reyes de la dinastía
Tudor Enrique VIII, María I e Isabel I, los Estuardo Jacobo I, Carlos I, Carlos II y María II, ésta última junto con su consorte Guillermo III de Orange. La ceremonia cada vez tenía más detractores por la diferente filiación o afinidad religiosa de los monarcas y de quienes los rodeaban. Guillermo, habiendo practicado el rito, terminó por discontinuarlo por considerarlo una práctica supersticiosa. A Guillermo lo sucedió Ana I, última reina de la dinastía Estuardo, que protagonizó el rito por última vez en forma simplificada el 27 de abril de 1714. Entre los enfermos que fueron tocados por Ana figura Samuel Johnson, el escritor, crítico literario y creador de un diccionario del idioma inglés. Él sufrió de escrófula en su infancia: cuando tenía 30 meses de edad, su madre premunida de un certificado médico de que el niño tenía efectivamente escrófula y que no había recibido el tacto real, lo llevó ante la reina. El procedimiento no tuvo efecto. Una intervención quirúrgica posterior en los ganglios le dejó una cicatriz en la parte baja de la cara y el cuello. Johnson llevó el ángel de oro colgado del cuello por el resto de su vida ${ }^{46}$.

Se ha afirmado que los reyes de diversas ramas de la dinastía de los Capeto que ejercieron la ceremonia en Francia desde el siglo XVI fueron los siguientes: Luis XII, Francisco I, Enrique II, Carlos IX, Enrique III, Enrique IV, Luis XIII, Luis XIV, Luis XV y Luis XVI; a estos se agrega Carlos $\mathrm{X}$ que fue el último en practicarlo. Presa de la indecisión y temiendo burlas de los liberales, tras una postergación tocó alrededor de 120 escrofulosos en un hospicio de Reims el 31 de mayo de 1825. Esto efectivamente le acarreó irónicos comentarios de la prensa. Las monjas del hospicio intentaron un seguimiento de los pacientes en un período de 3 meses y medio y estimaron que con seguridad se habían curado cinco de ellos ${ }^{47}$.

\section{Conclusiones}

La pretensión de detentar el don de sanación desplegado en una liturgia solemne con asistencia multitudinaria de enfermos y espectadores de todas las clases sociales habría pasado así a ser un elemento político. Por una parte, estimulaba la aceptación del concepto del origen y apoyo divino de la realeza; por otra, acrecentaba la admiración y credulidad del pueblo por la figura del rey, quien una y otra vez mostraba su solicitud por los pobres y enfermos.

El pretendido carácter divino hereditario del poder taumatúrgico de las generaciones de la realeza pudo lograrse solamente en Francia e Inglaterra, países relacionados por vínculos territoriales y familiares, donde la organización administrativa del gobierno y el entendimiento de los soberanos con las autoridades eclesiásticas contribuyeron a aceptar la creencia en el rito y su realización.

La elección de las escrófulas como objeto de la ceremonia de curación puede haberse debido a una alta 
frecuencia de ellas, a su aspecto evidente a la sola inspección o a rasgos de su evolución que pueden simular la curación. A diferencia de lo entendido en la época que se trataba de una sola enfermedad, algunas masas patológicas cervicales podían corresponder a afecciones graves; pero también a otras banales que pueden cursar con remisiones espontáneas.

Aparte de no encontrarse criterios estrictos en cuanto al diagnóstico de escrofulosis, comprensiblemente no se dispone tampoco de estudios confiables de seguimiento de la evolución de los enfermos tratados con el toque del rey. Los informes de las curaciones fueron, en general, escritos por funcionarios de la corte. La mayoría de los datos entresacados por los historiadores y el conocimiento actual de la linfoadenitis tuberculosa no permiten aceptar la idea de una curación milagrosa masiva.

\section{Resumen}

Desde el siglo XI y hasta principios del siglo XIX se desarrolló en Francia e Inglaterra una ceremonia por la cual los reyes, a los que se creía dotados de un don divino hereditario, por el tacto de su mano podían curar las escrófulas, nombre que ha correspondido a la linfoadenitis tuberculosa que afecta principalmente los ganglios del cuello. El rito llegó a celebrarse regularmente ante grupos de enfermos y una multitud de espectadores. La creencia en ese poder milagroso se basaba en que el monarca, por el hecho de ser ungido y coronado en una ceremonia religiosa, asumía un carácter sacerdotal junto con el poder temporal. Los monarcas de Francia e Inglaterra habrían aprovechado y estimulado la credulidad de sus súbditos para afianzarse frente al poder de los señores feudales y en momentos en que ocurría una alternancia de dinastías. La elección de la escrófula puede haberse debido a su alta frecuencia, a que puede evolucionar con fases interpretables como curación, y a que el concepto de escrófula pudo incluir otras lesiones con tendencia espontánea a episodios de remisión. Los datos históricos disponibles y el conocimiento actual de la linfoadenitis tuberculosa no sustentan la creencia en curaciones milagrosas masivas.

\section{Referencias bibliográficas}

1.- Porter R. The greatest benefit to mankind, a medical history of humanity. New York: W.W. Norton \& Company; 1997, p. 111.

2.- De Fleury H. Vie de Robert II le Pieux. Traducción de F. Guizot 1824. www.compusetampois.com/cls-11-helgaldusvitaroberti1824guizot.html (Consultado el 26 de enero de 2014).

3.- Barlow F. The King's evil. Engl Hist Rev 1980; 95: 3-27.

4.- Bloch M. Los comienzos del tacto de las escrófulas. Capítulo
1. Los reyes taumaturgos. México: Fondo de Cultura Económica; 1988, 35-56.

5.- Shakespeare W. La tragedia de Macbeth. Acto 4, escena 3. En: Obras completas, $13^{\mathrm{a}}$ edición Madrid: Aguilar Ediciones S. A. de Ediciones; 1965, p. 1614-5.

6.- Shakespeare W. The tragedy of Macbeth. The Oxford Shakespeare. Oxford: Oxford University Press; 1990, p. 188.

7.- $\quad$ Shakespeare W. Op cit, p. 67.

8.- $\quad$ San Isidro de Sevilla, Etimologías. Libro IV: Acerca de la Medicina. Madrid: Biblioteca de Autores Cristianos; 1982, p. 497.

9.- Bloch, M. Op cit. Los comienzos del tacto de la escrófula. Capítulo 1, p. 35-56.

10.- Biblia de Jerusalén. Marcos 16: 18. Bilbao: Desclée de Brouwer; 1998, p. 1493.

11.- Farquhar H. Royal Charities. Angel as healing-pieces for the King's evil. En: www.britnumsoc.org/publications/ Digital\%20BNJ/pdfs/1916_BNJ_12_6.pdf (Consultado el 3 de febrero de 2014).

12.- Farquhar H. Royal charities. II Touchpieces for the Kings Evil. BNJ 1917: 13: 95-163. www.britnumsoc.org/ publications/Digital\%20BNJ7pdfs/1917_BNJ_13_5.pdf (Consultado el 16 de febrero de 2014).

13.- Farquhar H. Royal charities III. Continuation of Touch pieces for the King's Evil. BNJ 1918: 14: 89-120.

14.- Grzybowski S, Allen EA. History and importance of scrofula. Lancet, 1995; 946 (8988): 1472-4.

15.- Wheeler S. Henry IV of France touching for scrofula, by Pierre Firens. J Hist Med Allied Sci, 2003; 58: 79-81.

16.- Evelyn J. The diary of John Evelyn. Vol I: New YorkLondon: Walter Dunne; 1901, p. 334.

17.- Rivera García A. La realeza medieval según Ernst $H$. Kantorowicz. www.saavedrafajardo.org/Archivos/ equipofilosofia/documento8.pdf (Consultado el 3 de febrero de 2014).

18.- Biblia de Jerusalén. Op cit. Levítico 8:12, p. 128.

19.- Biblia de Jerusalén. Op cit 1 Samuel 16:13, p. 334.

20.- Biblia de Jerusalén. 1 Reyes 1:39, p 382. Desclée de Brouwer, Bilbao 1998.

21.- Davis WS. A history of France; from the earliest times to the Treaty of Versailles. Cap 3: From Franks to Frenchmen. Boston: Houghton Mifflin Company; 1919. Versión digitalizada (2012).

22.- Rogelio de Howden. Chronica (1201). Orden de la coronación de Ricardo Corazón de León. En: El Renacimiento Europeo del siglo XII, textos y documentos, p. 15-17. Selección y traducción de JM Cerda. Santiago: Universidad Gabriela Mistral; 2012, p. 15-17.

23.- Bloch M. Op cit Capítulo 2: Los orígenes del poder de curación de los reyes: la realeza sagrada en los primeros siglos de la Edad Media, p. 57-87.

24.- Orlandis J. Breve historia del cristianismo. $2^{\mathrm{a}}$ Edición, Santiago. Editorial Universitaria; 1998, p. 82-83.

25.- Lenzenweger J, Stockmeier P, Amon K, Zinnhobler R. Historia de la Iglesia Católica. Barcelona: Editorial Herder; 1989 , p. 262-4.

26.- Bloch M. Op cit, Capítulo 1. Los comienzos del tacto de las escrófulas, p. 37-8.

27.- Tainmont J. A historical vignette (18). The King's evil: scrofula, physicians and the Royal touch. B-ENT, 2010; 6: 153-9. 
28.- Fraser R. The story of Britain; from the Romans to the present: a narrative History. Norman and Angevin. New York: W.W, Norton \& Company; 2003. Versión digitalizada.

29.- Bloch M. Op cit, p. 38

30.- Green J A. Henry I. King of England and Duke of Normandy. Cambridge, UK: Cambridge University Press; 2009, p. 138

31.- Fontanilla J M, Barnes A, Fordham von Reyn C. Current diagnosis and management of peripheral tuberculosis lymphadenitis. Clin Infect Dis, 2011; 53: 555-62.

32.- Dormandy T. The white death: a history of tuberculosis. Capítulo 29: Milk. London: The Hambledon Press; 1999, p. 329-38.

33.- Daniel TH. Captains of death; the story of tuberculosis. Capítulo 15: Bacille Calmette Guérin. Rochester: University of Rochester Press; 1999, p. 131-42.

34.- Koch R. The aetiology of tuberculosis. Am Rev Tuberc, 1932; 25: 285-323. Traducción del alemán de Berna y Max Pinner.

35.- Dormandy T. Op cit. Capítulo 1: The mists of History, p 4.

36.- Bynum H. Capítulo 2: All with 'a touch of consumption'? Spitting blood; the history of tuberculosis. Oxford: Oxford University Press; 2012 (Versión digitalizada).
37.- Wiseman R. A treatise of the King's evil. Major RH. Classic Descriptions of Disease, $3^{a}$ edición. Springfield 1982, p. 23946.

38.- Escrófulas. En: Diccionario de Ciencias Médicas, por una sociedad de los más célebres profesores de Europa. Traducido al castellano por varios Facultativos de esta Corte. Tomo XIII. Madrid: Imprenta de Don Mateo Repullés; 1823, p. 52-93.

39.- Jackson HJ. Coleridge on the King's Evil. Studies in Romanticism, 1977; 16: 337-42.

40.- Wiseman R. Op cit.

41.- Bloch M. Op cit Capítulo 8: Decadencia y muerte del tacto, p 347-68.

42.- Bloch M. Op cit. Capítulo 9: Los primeros ensayos de interpretación racionalista, p. 371-89.

43.- Pepys S. Diary and correspondence of Samuel Pepys FRS, $4^{\mathrm{a}}$ edición. London: Henry Colburn: 1854, p. 170.

44.- Wiseman R. Op cit.

45.- Bloch M. Op cit. Capítulo 9: Los primeros ensayos de interpretación racionalista, p. 371-89.

46.- McHenry LC, MacKeith R. Samuel Johnson childhood illnesses and the King's evil. Med Hist 1966; 10: 386-99.

47.- Bloch M. Op cit. Capítulo 7: El milagro real en la época de las luchas religiosas y del absolutismo, p. 285-389. 